\title{
Prevalence of Counseling Needs in Late Adolescent College Students of India
}

\author{
Suneetha Kandi \\ Andhra University, India \\ sunikandi@yahoo.com
}

\begin{abstract}
Prevalence of counseling needs of college students studying professional courses is assessed. 1038 (579 females; 459 males) students of 13 professional colleges (Engineering, Medicine \& Pharmacy) in two districts of Andhra Pradesh, India participated. Average age of the late adolescence sample is 18.24. Data is collected by purposive random sampling using specially designed tool Student Counseling Needs Questionnaire. Data was analyzed using Statistical Package for Social Sciences -16.0. Prevalence rates of counseling needs on five dimensions: academic, personality/adjustment, social, family and clinical are identified. Analysis of results indicated high prevalence rates in the academic dimension and low in the family dimension. The top five needs are to enhance communication skills, memory techniques, learning methods, attention/concentration, and work commitment. Significant gender differences are found.
\end{abstract}

Keywords: Counseling needs, college students, professional courses, Student Counseling Needs Questionnaire, late adolescents. 